\title{
Passenger pigeon genomic diversity and extinction
}

\author{
PW Hedrick ${ }^{1}$
}

Received: 12 January 2018 / Revised: 14 February 2018 / Accepted: 15 February 2018 / Published online: 12 March 2018

(c) The Genetics Society 2018

Understanding the factors that influence extinction and persistence of species is one of the key goals of genetical, evolutionary, and ecological aspects of conservation biology. Passenger pigeons (PP), perhaps the most abundant bird species ever with estimates of 3-5 billion birds, went extinct in 1914. This extinction was primarily the result of new and efficient hunting techniques introduced in the 19th century that included the new technology of the telegraph, which was used to inform hunters where the flocks were migrating and roosting, and the newly developed railroads, which provided transportation for the hunters and for shipping the killed birds to cities for food. Using current molecular analysis and in an effort to provide insight into this extinction, Murray et al. (2017) have now examined the impact of natural selection on the PP genome by comparing to its closest living relative, the band-tailed pigeon (BP).

Overall, Murray et al. (2017) found that the average diversity in PP was twice as high as that in BP (average nucleotide diversities $\pi$ of 0.08 in PP and 0.04 in BP). However, this difference was much less than that expected under a population genetics model if the population size of PP was several orders of magnitude larger than that of current BP population size estimates. Murray et al. (2017) concluded that this difference is the result of the "surprisingly pervasive influence of natural selection" and not past population instability as suggested by Hung et al. (2014).

Their most striking finding was the pattern of genetic diversity over the PP genome with quite high variation of diversity in different chromosomal regions compared to the very uniform diversity over the genome in BP (see Figure 2B in Murray et al. 2017). In fact, PP had nucleotide diversity that varied around 10 -fold between genomic

\section{PW Hedrick}

philip.hedrick@asu.edu

1 School of Biological Sciences, Arizona State University, Tempe, AZ 85287, USA regions with purported high recombination rates (the ends of the ten macrochromosomes and the microchromosomes) also having high nucleotide diversity around or approaching 0.02 , while genomic regions with low purported recombination rates (centers of the ten macrochromosomes) had low nucleotide diversity of around 0.002 .

The correlation of nucleotide diversity with recombination could be the result of several factors. First, it could be the result of selective sweeps of adaptive variants. Although selective sweeps of adaptive variants reduce diversity at nearby sites, positive selection is thought to be more efficient and important in regions of higher recombination and diversity (Charlesworth and Campos 2014). In support of positive selection, Murray et al. (2017) identified 32 genes that showed the evidence of adaptive evolution in PP, some of which they suggested were adaptive to living in high density. They also provided some evidence that has been a faster rate of adaptive evolution in PP than in BP. Second, the positive association of diversity and recombination rates is also expected to occur when there is purifying selection against deleterious variants (Charlesworth and Campos 2014). Finally, Murray et al. (2017) also suggested that biased gene conversion rates are expected to be higher in regions with higher recombination. Murray et al. (2017) provided evidence that all three factors were significant but it would be important to know how much contribution each of these factors make to the extreme variation in nucleotide diversity over the PP genome.

The most recognized genetic reason for extinction risk is inbreeding depression and there are many examples of high inbreeding depression in bird species, for example, in the great tit (Szulkin et al. 2007), collared flycatchers (Kruuk et al. 2002), and house sparrows (Jensen et al. 2007). The recent number of documented cases of large inbreeding depression appears partly due to the examination of additional fitness components besides viability, such as fecundity and mating success, and partly due to measuring inbreeding depression in more stressful natural environments. For example in red deer, which has large population numbers, given an inbreeding level equivalent to that in 
progeny from a mating between half sibs, the predicted lifetime breeding success was only about $10 \%$ that when there was no inbreeding (Huisman et al. 2016).

The number of lethal equivalents, which measures the potential for inbreeding depression, is expected to increase with a larger population size (Garcia-Dorado 2012; Hedrick and Garcia-Dorado 2016). This increase in inbreeding load occurs because the expected number of lethal equivalents is determined by the mutation-selection-genetic drift balance, which assumes that mutation produces genetic variants with detrimental effects and both purifying selection and genetic drift reduce this variation (Garcia-Dorado 2012; Hedrick and Garcia-Dorado 2016). Therefore, lower inbreeding load would be predicted for smaller populations, while larger populations are expected to experience more severe fitness reduction under inbreeding because they have more standing detrimental genetic variation. In other words, PP could have very well suffered high inbreeding depression when their numbers were reduced from very high levels to very low levels. In fact, there was one captive flock alive when the last PP in the wild disappeared but it was already highly inbred, the individuals were old, and it died out (Revive\&Restore 2017). Documentation of the presence and size of genomic runs of homozygosity in PP would provide information about the level of recent inbreeding and potentially genomic regions to examine for inbreeding depression.

The most similar, concurrent example is that of the plains bison, which also had very large population sizes with total numbers estimated to be about 60 million individuals. They were hunted to near extinction in North America during much the same period in the late 19th century as the PP. However, bison survived this extreme hunting because a few were protected in remnant populations on five private ranches and what is now known as Yellowstone National Park. They were subsequently gradually increased from less than 100 founders to around 20,000 now in conservation herds (Hedrick 2009). In other words, although there are genetic concerns about present-day plains bison, including extreme inbreeding depression in a captive herd (Halbert et al. 2004, 2005; Hedrick 2009), the near extinction of bison is not thought to be related to past natural selection (Hedrick 2009).

Recent interest in the PP has been increased by a proposal to revive PP from extinction using a gene-edited BP genome (Revive \& Restore 2017). Although Murray et al. (2017) did not discuss application of their results to this deextinction proposal, one focus in such a project could be on changing regions that have fixed differences between PP and BP identified by them, particularly for genes that show evidence of adaptive evolution in PP. Another potential object could be to focus on the regions of high diversity in the PP to provide genetic variation for future adaptation. However, gene-editing the BP genome to produce PP genes might create unpredictable results in the genetic background of BP, a species with quite different ecology and behavior and a species that is declining about 3\% annually (Keppie and Braun 2000). In addition when considering such proposals, other concerns about de-extinction of the PP, including a number of behavioral, ecological, environmental, and political issues, need to be addressed.

Murray et al. (2017) concluded that "that passenger pigeons may have evolved traits that were adaptive when their population was large but that made it more difficult for them to survive after their population was diminished by the commercial harvest. More broadly, our results suggest that even species with large and stable population sizes can be at risk of extinction after a sudden environmental change." In other words, Murray et al. (2017) suggested that PP could not adapt to the environmental effects related to the low population numbers present after its decimation from hunting. However, there is generally insufficient information about reproduction and survival in PP populations at different densities and conditions to evaluate properly important aspects of this hypothesis. Further, Revive\&Restore (2017) stated that PP "were observed breeding in single pairs and flocks of dozens during the 1800s" and then concluded that PP "reproductive rate was not affected by low numbers and flock fragmentation" (see also Roberts et al. 2017). In other words, it appears that overhunting was the cause of the extinction of PP and unclear whether low genomic diversity and/or PP adaptation to large numbers played a significant role in PP extinction. Further, given limited knowledge about the PP and its extinction it also seems that it is not a good candidate species to use to understand extinction processes in other species with large and stable population sizes.

Overall, the analysis of Murray et al. (2017) provides a strong foundation for examination of genomic data in the iconic PP and its relative BP. As discussed above, these results suggest that understanding of the basis of the extreme variation in nucleotide diversity over the genome, the potential contribution of inbreeding depression, the impact of these results on proposals to resurrect the PP, and the potential role of adaptation to large numbers in extinction, are all avenues for further examination.

Acknowledgments I am grateful to Brian Charlesworth, Marty Kardos, and two reviewers for the comments on the earlier versions of the manuscript.

\section{Compliance with ethical standards}

Conflict of Interest The authors declare that they have no conflict of interest. 


\section{References}

Charlesworth B, Campos JL (2014) The relations between recombination rate and patterns of molecular variation and evolution in Drosophila. Ann Rev Genet 48:383-403

Garcia-Dorado A (2012) Understanding and predicting the fitness decline of shrunk populations: inbreeding, purging, mutation, and standard selection. Genetics 190:1461-1476

Halbert ND, Raudsepp T, Chowdhary BP, Derr JN (2004) Conservation genetic analysis of the Texas State Bison Herd. J Mammal 85:934-931

Halbert ND, Grant WE, Derr JN (2005) Genetic and demographic consequences on importing animals into a small population: a simulation model of the Texas State Bison Herd (USA). Ecol Model 181:263-286

Hedrick PW (2009) Conservation genetics and North American bison (Bison bison). J Hered 100:411-420

Hedrick PW, Garcia-Dorado A (2016) Understanding inbreeding depression, purging, and genetic rescue. Trends Ecol Evol 31:940-952

Huisman J, Kruuk LEB, Ellis PA, Clutton-Brock T, Pemberton JM (2016) Inbreeding depression across the lifespan in a wild mammal population. Proc Natl Acad Sci USA 113:3585-3590
Hung C-M, Shaner P-JL, Zink RM, Liu W-C, Chu T-C et al. (2014) Drastic population fluctuations explain the rapid extinction of the passenger pigeon. Proc Natl Acad Sci USA 111:10636-10641

Keppie DM, Braun CE (2000) Band-tailed Pigeon (Patagioenas fasciata), version 2.0. In: Rodewald PG (ed) The Birds of North America. Cornell Lab of Ornithology, Ithaca, NY

Kruuk LE, Sheldon BC, Merila J (2002) Severe inbreeding depression in collared flycatchers (Ficedula albicollis). Proc Roy Soc Lond B 269:1581-1589

Jensen H, Bremset EM, Ringsby TH, Saether B-E (2007) Multilocus heterozygosity and inbreeding depression in an insular house sparrow metapopulation. Mol Ecol 16:4066-4078

Murray GGR, Soares AER, Novak BJ, Schaefer NK, Cahill JA, Baker AJ et al. (2017) Natural selection shaped the rise and fall of passenger pigeon genomic diversity. Science 358:951-954

Revive\&Restore (2017) Passenger Pigeon Project. http://reviverestore. org/about-the-passenger-pigeon/

Roberts DL, Jarić I, Solow R (2017) On the functional extinction of the passenger pigeon. Cons Biol 31:1192-1195

Szulkin M, Garant D, MeCleery RH, Sheldon BC (2007) Inbreeding depression along a life-history continuum in the great tit. J Evol Biol 20:1531-1543 